\title{
Obstetrical outcomes after vaginal repair of caesarean scar diverticula in reproductive- aged women
}

\author{
Xingchen Zhou', Xiaogian Yang ${ }^{2}$, Huihui Chen ${ }^{1}$, Xuhong Fang ${ }^{1 *}$ and Xipeng Wang ${ }^{1 *}$ (D)
}

\begin{abstract}
Background: Although vaginal repair has been conducted to manage caesarean scar diverticula, most studies evaluated only the gynaecological outcomes post-surgery, and their obstetrical outcomes were unknown. This study aimed to evaluate the obstetrical outcomes in vaginal repair-treated caesarean scar diverticula patients.

Methods: A series of 51 symptomatic women with caesarean scar defects or a thickness of the remaining muscular layer of less than $3 \mathrm{~mm}$ according to transvaginal ultrasound were included. We retrospectively evaluated the gynaecological and obstetrical outcomes after vaginal repair and histologically analysed the defect.

Results: Transvaginal ultrasound revealed that the thickness of the remaining muscular layer significantly increased from $2.24 \pm 0.81 \mathrm{~mm}$ to $6.10 \pm 1.43 \mathrm{~mm} 3$ months after vaginal repair. The duration of menstruation significantly decreased from $14.29 \pm 3.13$ days to $8.31 \pm 2.14$ days post-vaginal repair. Notably, 26 of the 51 (50.98\%) women who were followed for more than 15.04 months post-surgery achieved pregnancy. A total of 6 of the 26 pregnancies (23.08\%) resulted in miscarriages, including 5 early miscarriages and 1 late miscarriage. Among the 20 women who achieved pregnancy without miscarriage, 18 had term deliveries, 2 had preterm birth, and none reported uterine rupture. Histological analysis was performed in all 51 cases. Muscle fibre density was significantly lower in the scar than in the myometrium adjacent to the scar and collagen expression was markedly increased in the scar tissue.
\end{abstract}

Conclusion: Satisfactory gynaecological and subsequent obstetrical outcomes can be achieved in vaginal repairtreated caesarean scar diverticula patients.

Keywords: Caesarean scar defect, Vaginal repair, Prolonged menstrual bleeding, The thickness of the remaining muscular layer, Obstetrical outcomes

\section{Background}

In China, the proportion of caesarean sections (CSs) performed in 2010 was $35-58 \%$, which has attracted significant concern regarding the development of caesarean scar diverticula (CSD) [1]. CSD are reservoir-like pouch defects at the site of a previous caesarean incision and result from incomplete scar healing on the anterior wall of the uterine isthmus (Fig. 1) [2]. It has been suggested that the incidence of CSD is as high as $61 \%$ after one CS

\footnotetext{
* Correspondence: fangxuhong@xinhuamed.com.cn; wangxipeng@xinhuamed.com.cn

Xingchen Zhou and Xiaoqian Yang are co-first authors.

${ }^{1}$ Department of Gynecology, Xinhua Hospital affiliated with Shanghai Jiaotong University, 1665 Kong Jiang Rd, Yang Pu District, Shanghai 200092, China

Full list of author information is available at the end of the article
}

and reaches $100 \%$ after at least three CSs [3]. With the increasing CS rate and the two-child policy implemented in China, the complications of CSD, such as prolonged menstrual bleeding, secondary infertility, and even uterine rupture during a subsequent pregnancy, have emerged as important clinical problems [4]. The prevalence of CSD-associated prolonged menstrual bleeding ranges from 63.6 to $88 \%$ [5]. The exact pathogenesis responsible for CSD has not been fully elucidated. Multiple CS procedures, a retroflexed uterus, cervical dilation, the type and technique of uterine closure, and maternal age at the time of caesarean section were suggested to contribute to the potential risk for CSD [2, 6-8].

Clinical guidelines have yet to be established for the management of CSD. CSD can be treated using a 


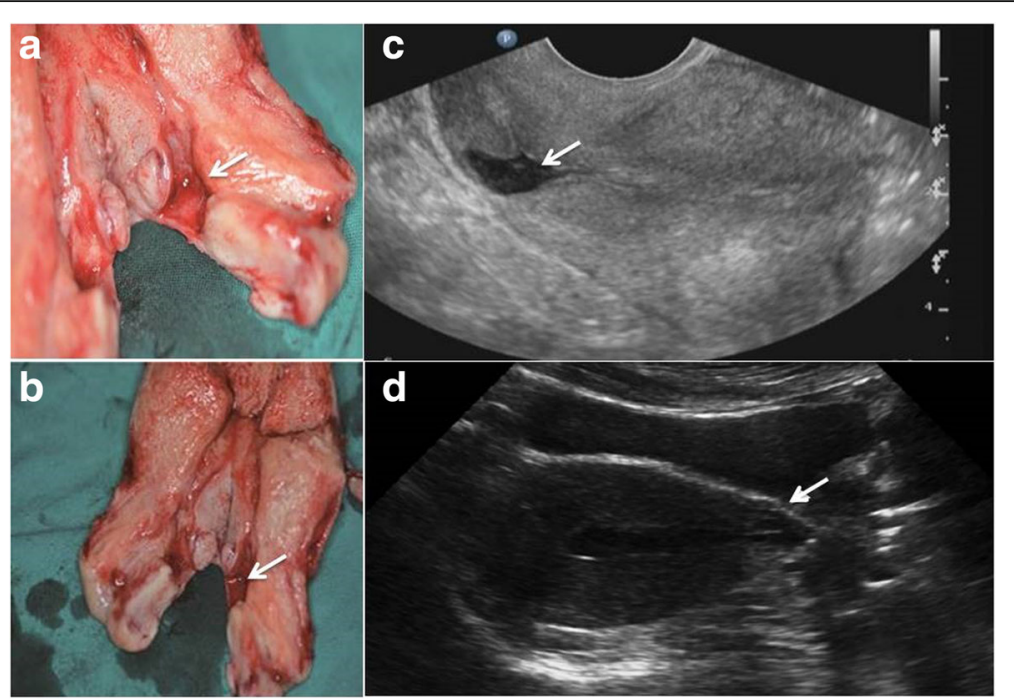

Fig. 1 The shape of CSD. $\mathbf{a}, \mathbf{b}$ : A section view of a hysterectomy specimen. A deep anterior defect covered with a thin layer of myometrium (white arrow) can be observed at the level of the supposed site of CS. c, d: The white arrows indicate the shape of CSD under transabdominal and transvaginal ultrasonography

hysteroscopy transcervical approach, laparoscopic abdominal approach, and transvaginal surgical repair. Above all, vaginal repair has been identified by our group and other researchers as a less invasive, safe and effective surgical procedure to treat CSD [9-11]. Vaginal repair can not only resolve CSD-associated prolonged menstrual bleeding but can also restore the anatomical structure in patients with CSD. A retrospective review reported that the improvement in menstruation can reach 93.5\% (43/46) in CSD patients who received vaginal repair [9]. Since April 2013, our team has performed vaginal repair to treat CSD. Our previous studies reported that $80.3 \%(94 / 117)$ of CSD patients experienced a duration of menstruation $\leq 10$ days, and the thickness of the remaining muscular layer (TRM) exhibited significant improvements $(2.56 \pm 1.32$ vs $8.65 \pm 3.11, \quad P<0.001)$ at 6 months post-surgery [10]. The results of our other study revealed that $60.0 \%(48 / 80)$ of the patients with CSD that resolved after surgery achieved a duration of menstruation $\leq 7$ days at the median follow-up (11.28 months) [11]. Therefore, we believe that vaginal repair can benefit women who experienced CSD and want to achieve pregnancy again.

However, the subsequent pregnancy outcome in vaginal repair-treated women was not included in our previous studies. To address this deficiency, women who had undergone vaginal repair of CSD, whose TRM was less than $3 \mathrm{~mm}$ and who attempted pregnancy were followed long term in our institution. Their clinical characteristics were analysed, and obstetrical outcomes were recorded.

\section{Methods}

\section{Patient population}

A total of 340 CSD patients were managed using vaginal repair between April 2013 and May 2016 at Shanghai
First Maternity and Infant Hospital, Tongji University. he presence of remaining myometrium (TRM) was determined by transvaginal ultrasound (TVU), which was clearly described in our previous studies [10,11] To measure the thickness of the residual muscle layer of the diverticulum, the patient's should be a cursor was placed at the interface between the uterine and the bladder wall, and another cursor was positioned at the bottom of the CSD. Three different measurements of length, width, height, depth and TRM were obtained. All of the patients underwent TVU to evaluate the parameters of the CSD preoperatively and postoperatively, namely, CSD length, width, depth and TRM. Among the enrolled participants, 51 patients attempted to become pregnant; their clinical characteristics are summarized in Table 1. Our present retrospective study was approved by the Ethics Committee of Shanghai First Maternity and Infant Hospital (KS1512). Informed written consent was obtained from all participants after they received clear information about the procedure and possible surgical outcomes of vaginal repair.

\section{Surgical technique}

The complete surgical technique was described in detail in our previous studies and has been recognized as an effective approach for anatomic correction of CSD as well as symptomatic relief of prolonged menstrual duration [11]. The most important steps of vaginal repair of CSD are summarized again here. Briefly, the bladder was carefully dissected away from the uterus towards the abdominal cavity to open the vesicovaginal space and reach the peritoneum. CSD tissue was easily found once the abdominal cavity was entered, and the lower uterine 
Table 1 Clinical characteristics of the patients with CSD

\begin{tabular}{|c|c|}
\hline Characteristic & CSD patients $(n=51)$ \\
\hline Age, y & $31.25 \pm 3.36(24-42)$ \\
\hline \multicolumn{2}{|l|}{ Menstruation, d } \\
\hline Before VR & $14.29 \pm 3.13(8-25)$ \\
\hline After VR & $8.31 \pm 2.14(5-14)$ \\
\hline \multicolumn{2}{|l|}{ Uterus position, \% } \\
\hline Anteflexion & $37.3 \%(19 / 51)$ \\
\hline Retroflexion & $62.7 \%(32 / 51)$ \\
\hline Number of previous caesarean sections & $1.04 \pm 0.20$ \\
\hline One, \% & $96.1 \%(49 / 51)$ \\
\hline More than one, \% & $3.9 \%(2 / 51)$ \\
\hline \multicolumn{2}{|l|}{ CSD parameters before $\mathrm{VR}, \mathrm{mm}$} \\
\hline CSD length & $7.77 \pm 3.39(2-16)$ \\
\hline CSD width & $12.00 \pm 5.42(4-27)$ \\
\hline CSD depth & $6.32 \pm 2.45(2-12)$ \\
\hline TRM, mm & $2.24 \pm 0.81(0.7-4.6)$ \\
\hline Postoperative TRM, mm & $8.36 \pm 2.96(3.0-13.9)$ \\
\hline CSD resolved after VR, \% & $68.63 \%(35 / 51)$ \\
\hline Persistent CSD after VR, \% & $31.37 \%(16 / 51)$ \\
\hline \multicolumn{2}{|l|}{ Persistent CSD parameters, mm } \\
\hline CSD length & $5.78 \pm 2.64(2-11)$ \\
\hline CSD width & $9.00 \pm 5.32(3-20)$ \\
\hline CSD depth & $4.33 \pm 1.00(3-6)$ \\
\hline TRM, mm & $6.10 \pm 1.43(3.8-8.1)$ \\
\hline Pregnancy rate, \% & $50.98 \%(26 / 51)$ \\
\hline Spontaneous abortion rate, \% & $23.08 \%(6 / 26)$ \\
\hline Median follow-up time, months & $28.42 \pm 9.74(15.04-50.46)$ \\
\hline
\end{tabular}

Data are presented as the means \pm SD or percentages

Note: $\mathrm{CSD}=$ caesarean scar diverticula; VR = vaginal repair of CSD; TRM = thickness of the remaining muscular layer

segment was completely exposed. The CSD tissue was then thoroughly removed. All surgical procedures reported in the current series were performed by the same surgeon. All patients were discharged from the hospital on postoperative day 3. After the subsequent TVU at 3 months post-surgery, the women were told they could attempt pregnancy starting 1 year post-surgery if their TRM had reached a thickness of more than $3 \mathrm{~mm}$.

\section{Data collection and follow-up}

Preoperative and postoperative clinical information was gathered, including age; uterus position; reproductive history; menstrual duration; CSD length, width, and depth; and TRM. The follow-up was scheduled at 3 months after surgery for all patients, at which time a TVU was performed. The patients with a postoperative TRM more than $3 \mathrm{~mm}$ and a subsequent pregnancy attempt were recorded, and long-term follow-up was conducted. Their gestational age, pregnancy complications, interval from vaginal repair to pregnancy, neonatal birth weight, and infant sex were collected.

\section{Histology}

Histology was performed on tissues from all patients, and histochemical and Masson's staining were performed on the tissues of the 51 patients who attempted to become pregnant post-surgery to evaluate the muscle or collagen density of the remaining myometrium. Two areas were evaluated: the myometrium covering the scar and the myometrium directly adjacent to the scar. Haematoxylin-eosin staining was used for histology. Masson's staining was used to detect collagen. Panoramic viewer software was applied for histochemical quantification and morphological analysis.

\section{Statistical analysis}

Data are presented as the means \pm SD or percentage when appropriate. A paired t-test was used to analyse preoperative and postoperative data. Statistical analysis was performed using SPSS software (version 22, IBM Co., Armonk, NY, USA). Statistical significance was set at $P<0.05$.

\section{Results \\ Gynaecological outcomes}

The background characteristics of the women with CSD who attempted pregnancy after vaginal repair are shown in Table 1. Three months after the surgical procedure, the TRM dramatically $(P<0.001)$ recovered from $2.24 \pm$ $0.81 \mathrm{~mm}$ (range, $0.7-4.6$ ) to $8.36 \pm 2.96 \mathrm{~mm}$ (range, 3.013.9) on TVU. The menstrual duration significantly decreased $(P<0.001)$ from $14.29 \pm 3.13$ days to $8.31 \pm 2.14$ days 3 months after vaginal repair. CSD disappeared in $68.63 \%$ of patients $(35 / 51)$ at the 3-month follow-up. We present the clinical characteristics of the women with vaginal repair-treated CSD who achieved pregnancy without miscarriage in Table 2. A total of 19 women underwent one CS, and one woman underwent two CSs before vaginal repair of the CSD. The uterine positions were anteflexion in 11 women and retroflexion in 9 women. TVU revealed that the TRM significantly $(P<0.001)$ increased from $2.42 \pm$ $1.04 \mathrm{~mm}$ (range, 1.0-4.6) to $8.36 \pm 3.11 \mathrm{~mm}$ (range, 3.812.0) after the vaginal surgical procedure at the 3-month follow-up. The duration of menstruation significantly decreased $(P<0.001)$ from $14.20 \pm 2.76$ days to $8.00 \pm 2.19$ days post-vaginal repair. At the 3-month follow-up, 14 of the 20 (70.0\%) women had no evidence of CSD as measured by TVU. The interval from vaginal repair to pregnancy was $32.06 \pm 10.09$ months (range, 16.88-50.36).

\section{Obstetrical outcomes}

The obstetrical outcome of pregnancy was assessed in 26 of the 51 women (50.98\%) who were followed for 
Table 2 Clinical characteristics of the women who achieved pregnancy without miscarriage

\begin{tabular}{|c|c|}
\hline Characteristic & CSD patients $(n=20)$ \\
\hline Age, y & $31.00 \pm 3.06(26-38)$ \\
\hline \multicolumn{2}{|l|}{ Menstruation, $d$} \\
\hline Before VR & $14.2 \pm 2.76(8-20)$ \\
\hline After VR & $8.00 \pm 2.19(5-13)$ \\
\hline \multicolumn{2}{|l|}{ Uterus position, \% } \\
\hline Anteflexion & $55.0 \%(11 / 20)$ \\
\hline Retroflexion & $45.0 \%(9 / 20)$ \\
\hline Number of C-section deliveries & $1.05 \pm 0.22$ \\
\hline One, \% & $95.0 \%(19 / 20)$ \\
\hline More than one, \% & $5.0 \%(1 / 20)$ \\
\hline \multicolumn{2}{|l|}{ CSD parameters before VR, $\mathrm{mm}$} \\
\hline CSD length & $7.21 \pm 3.14(2-13)$ \\
\hline CSD width & $11.74 \pm 5.61(4-20)$ \\
\hline CSD depth & $6.53 \pm 2.65(2-11)$ \\
\hline $\mathrm{TRM}, \mathrm{mm}$ & $2.42 \pm 1.04(1.0-4.6)$ \\
\hline Postoperative TRM, mm & $8.36 \pm 3.11(3.8-12.0)$ \\
\hline CSD resolved after VR, \% & $70.0 \%(14 / 20)$ \\
\hline Persistent CSD after VR, \% & $30.0 \%(6 / 20)$ \\
\hline \multicolumn{2}{|l|}{ Persistent CSD parameters, mm } \\
\hline CSD length & $5.5 \pm 2.12(4-7)$ \\
\hline CSD width & $6.5 \pm 4.95(3-10)$ \\
\hline CSD depth & $4.5 \pm 0.71(4-5)$ \\
\hline TRM, mm & $4.95 \pm 1.48(3.9-6.0)$ \\
\hline $\begin{array}{l}\text { Interval from VR to pregnancy, } \\
\text { months }\end{array}$ & $32.06 \pm 10.09(16.88-50.36)$ \\
\hline Gestational age (days) & $266.03 \pm 6.44(245-273)$ \\
\hline Preterm birth rate, $\%$ & $10 \%(2 / 20)$ \\
\hline Neonatal birth weight, $g$ & $3149.75 \pm 308.24(2500-3850)$ \\
\hline Apgar score (5 min) & 10 \\
\hline Pregnancy complication rate, \% & $20.0 \%(4 / 20)$ \\
\hline Follow-up time, months & $32.06 \pm 10.09(16.88-50.36)$ \\
\hline
\end{tabular}

Data are presented as the means \pm SD or number (percentage) Note: CSD caesarean scar diverticula, C-section caesarean section, $V R$ vaginal repair of CSD, TRM thickness of the remaining muscular layer, $M$ male, $F$ female

more than 15.04 months post-surgery. Unfortunately, 6 of 26 pregnancies (23.08\%) resulted in miscarriages, which included 5 early miscarriages and 1 late miscarriage (Tables 1 and 2). None of the women attempted to deliver vaginally, and no cases of threatened uterine rupture or uterine rupture were detected in women who had successful births. As shown in Table 2, the mean gestational age was $266.03 \pm 6.44$ days, and the mean neonatal birth weight was $3149.75 \pm 308.24 \mathrm{~g}$ among the women who gave birth (Table 2). The detailed outcomes after vaginal repair of CSD in reproductive-aged women are shown in Table 3. Among the 20 women who achieved pregnancy without miscarriage, 18 had term deliveries, and 2 had preterm deliveries during the long-term follow-up period (32.06 \pm 10.09 months). In the women who had preterm deliveries, one woman gave birth at 35 weeks of gestation because of foetal heart rate decelerations on a non-stress test at a routine pregnancy check-up and threatened preterm labour. Another woman gave birth at $36+6$ weeks of gestation due to preterm premature rupture of the membranes. This woman haemorrhaged $945 \mathrm{ml}$ during the caesarean section. Four women who achieved term deliveries experienced pregnancy complications, including 2 women with gestational diabetes mellitus (GDM), 1 woman with placenta previa, and 1 woman with GDM as well as hypothyroidism during pregnancy (Table 3). However, all 20 women delivered healthy babies (Fig. 2).

\section{Histology}

Histological analysis was performed in all 51 cases. The muscular density of the residual myometrium covering the scar (Fig. 3a and b) was found to be significantly lower $(P<0.005)$ than that directly adjacent to the scar. Moreover, Masson's staining showed that the expression of collagen in CSD was $71.37 \pm 19.82 \%$ compared to $52.75 \pm 17.33 \%$ in healthy myometrium (Fig.3b). The collagen expression significantly increased in the scar defect (Fig. 3c and d).

\section{Discussion}

The most important clinical problems related to CSD are prolonged menstrual bleeding and adverse events that may affect subsequent pregnancies, including infertility, miscarriage, and uterine rupture. The prevalence of CSD-associated prolonged menstrual bleeding ranges from 63.6 to $88 \%$ [5]. Although the risk with CSD remains unclear, there is an obvious association between TRM and uterine rupture or dehiscence in subsequent pregnancies $[12,13]$. Therefore, repairing the CSD, especially rebuilding the muscular layer of the scar site, might control the symptoms of prolonged menstrual bleeding and decrease the risk of uterine rupture caused by CSD. Hysteroscopy niche resection, laparoscopic repair, laparoscopic-assisted vaginal repair, and vaginal repair are currently employed to treat CSD-related symptoms [1, 14].

Vaginal repair is effective for improving the menstrual duration in patients with CSD. An improvement in uterine bleeding after vaginal repair occurs in $80.3 \%$ to $93.5 \%$ of cases. A retrospective review reported that the improvements in menstruation can reach 93.5\% (43/46) in CSD patients who undergo vaginal repair [9]. Another study revealed that $92.9 \%(39 / 42)$ of vaginal repair-treated CSD 


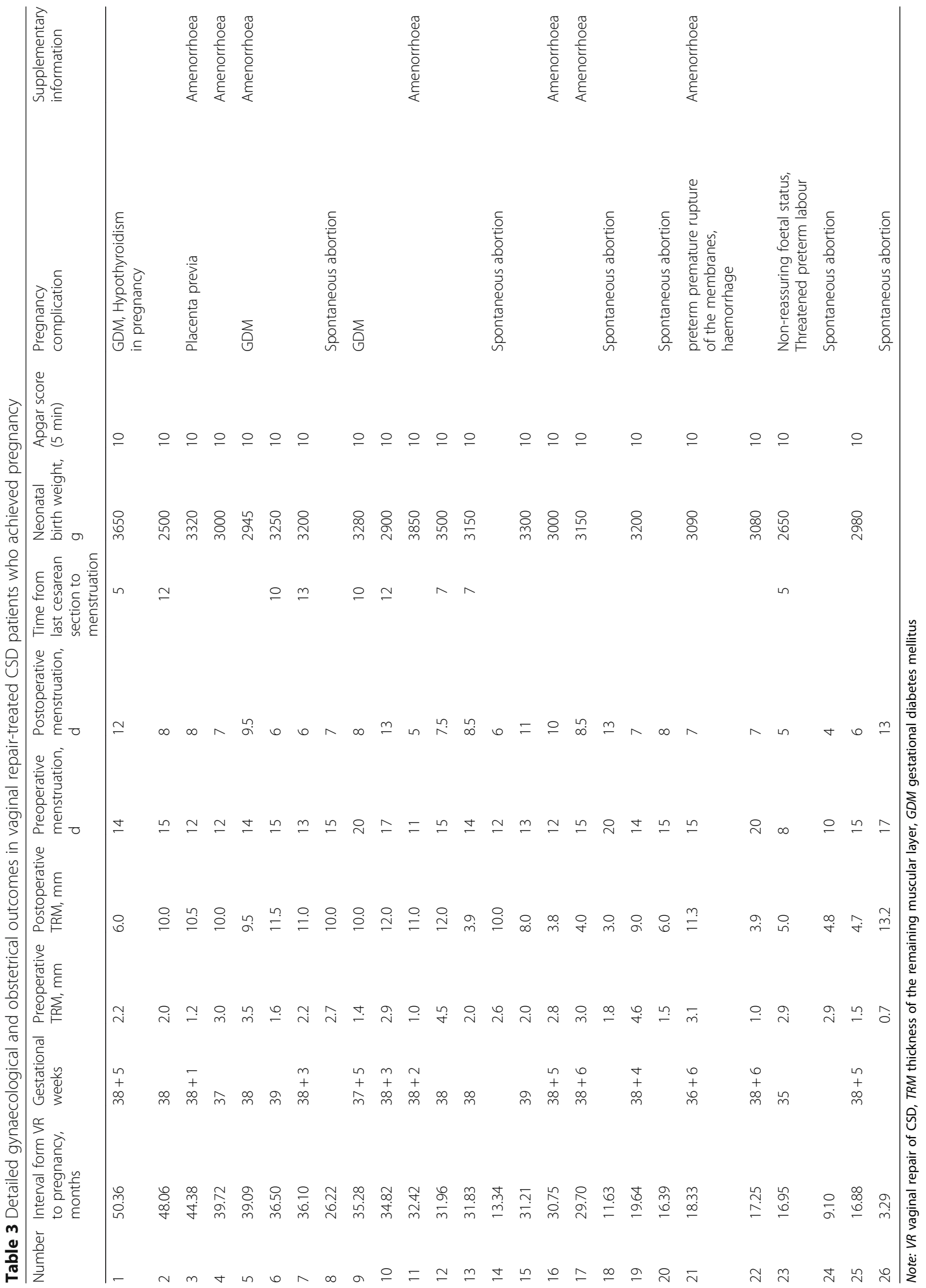




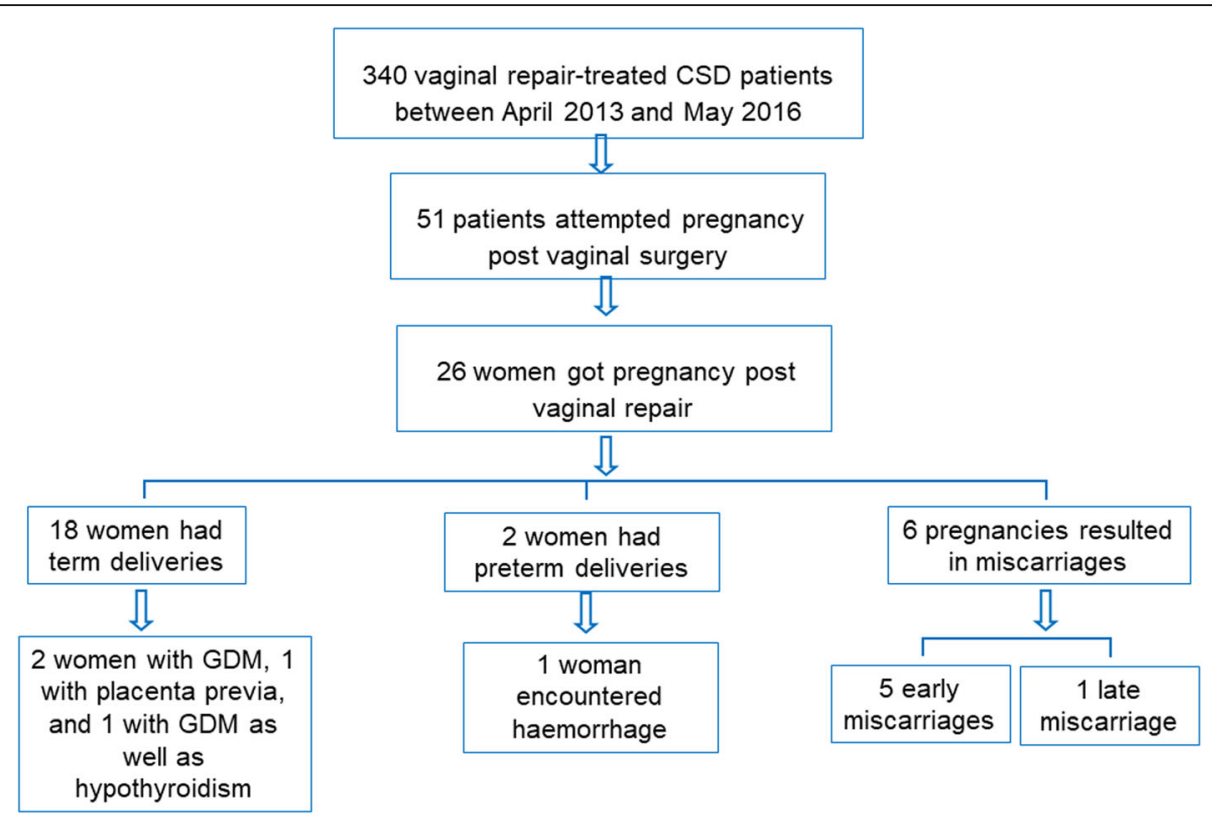

Fig. 2 The obstetrical outcomes after vaginal repair of CSD

patients reported significant improvements in menstruation at follow-up [15]. Our previous studies showed that 80.3\% (94/117) of vaginal repair-treated CSD patients experienced a menstrual duration $\leq 10$ days [10]. We also reported that the defect width of the preoperative CSD was the best prognostic index of the CSD anatomical repair effect post-vaginal repair [11]. The prognostic factors for the recovery of normal menstrual duration in CSD vaginal repair-treated CSD patients were assessed in the following study.
Although a series of studies have been conducted to investigate the management of CSD, most of the studies evaluated only the gynaecological outcomes post-surgery, and their obstetrical outcomes were unknown. Moreover, to date, no studies have compared obstetrical outcomes of laparoscopic repair and vaginal repair, and there are no guidelines for the selection of operative strategies for women with CSD who desire to become pregnant post-surgery. It has been reported that pregnancies occurred in 14 of 27 (51.9\%) women who underwent
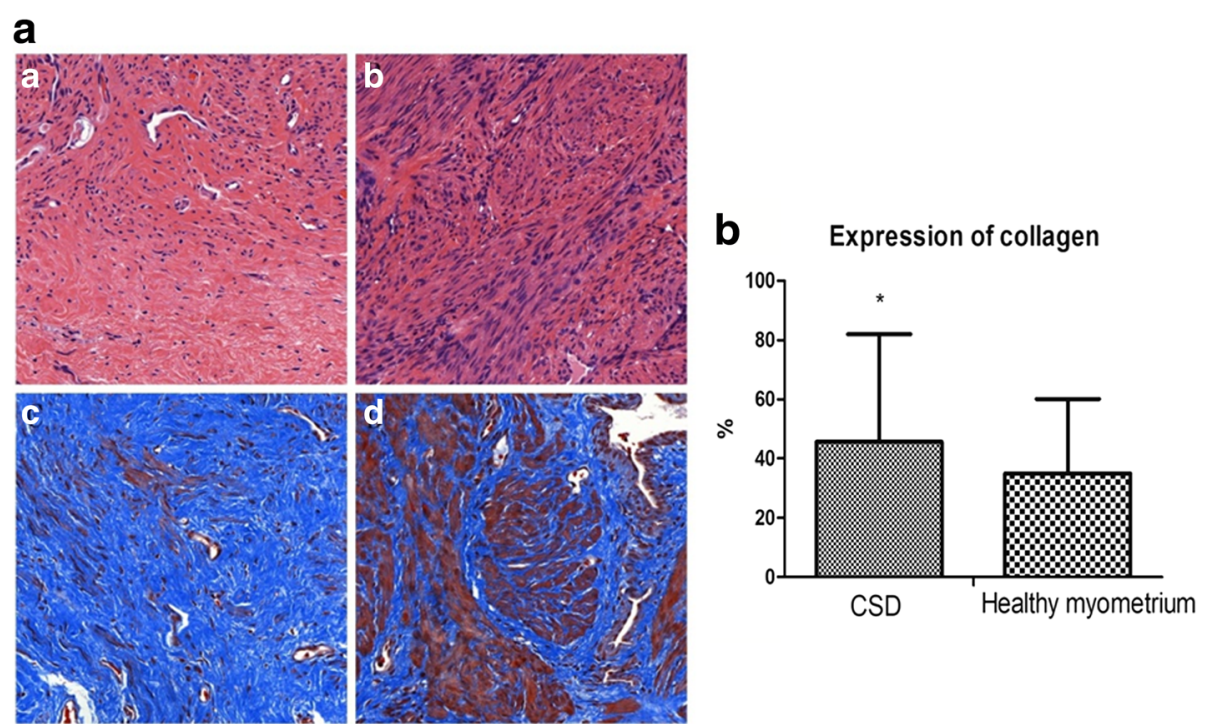

Fig. 3 Haematoxylin-eosin staining was used for histology ( $\times 100)$. a: Scar tissue defect. B: Tissue without defect adjacent to the scar; Masson's staining was used for collagen $(\times 100)$. C: Scar tissue defect. $\mathbf{d}$ : Tissue without defect adjacent to the scar. b: Histogram of the expression of collagen 
laparoscopic surgery $[16,17]$. In another study, 10 of 18 (55.6\%) women who were treated by laparoscopic repair achieved pregnancy following surgery [18]. The findings from an observational study showed that $44.4 \%$ (8/18) of women with infertility conceived and subsequently delivered their new-borns via CS after laparoscopic repair [1]. A total of $50.98 \%$ of the women in our study achieved pregnancy after vaginal repair, which indicates that vaginal repair is effective for improving fertility in patients with CSD. CS may lead to reduced fertility and prolonged inter-pregnancy intervals compared with vaginal deliveries. A meta-analysis conducted by Gurol-Urganci et al. suggests that the probability of future pregnancy and birth in women who have had a CS was 9\% lower and $11 \%$ lower, respectively, than that in women who delivered vaginally [19]. The median inter-pregnancy intervals after CS were 2-6 months longer than those after vaginal delivery [19]. A meta-analysis that included 85,728 women in whom CS was previously performed found the rate of subsequent pregnancy to be reduced by $10 \%$ compared with that in women who had vaginal deliveries [20]. Even if the precise pathophysiology remains unclear, incomplete uterine healing and postoperative infection may contribute to the pathophysiological reasons underlying the above phenomenon [21]. The rate of infertility caused by CSD has not been elucidated. It is reasonable to speculate that the rate of infertility in women with CSD was higher than that in women who had undergone CS. The accumulation of mucus or blood in the defect, which leads to the presence of intrauterine fluid, could prevent the penetration of sperm cells, endometrial receptivity, or embryo implantation, which may be responsible for the infertility caused by CSD $[1,18]$. In our study, $49.02 \%$ of women still did not achieve pregnancy.

Miscarriage was the most common adverse pregnancy outcome in 26 women with recognized pregnancy and previous CSD. Six women (6/26) suffered miscarriage in different stages of pregnancy. The underlying mechanisms of the association between CSD and miscarriage are unclear, and this adverse event may occur with no obvious underlying cause. Some studies report that the rate of spontaneous miscarriage ranges between 10 and $15 \%$ of recognized pregnancies [22]. Another study showed that miscarriage occurred in one in five pregnancies in women aged 31-36 years [21]. This number is greater in vaginal repair-treated CSD patients, with a rate of $23.08 \%$ reported in the current study. We believe that the miscarriage rate was correlated with age, and the relationship among CSD, miscarriage, and subsequent fertility warrants further investigation.

Uterine rupture is the most feared complication during pregnancy and labour in women who had previous deliveries via CS. TRM in the lower uterine segment (LUS) is a widely accepted indicator for the prediction of uterine rupture during labour. A full thickness of $3.0 \mathrm{~mm}$ determined via LUS can be considered a cutoff value to identify women at risk of uterine rupture, with a specificity of $85 \%$ and sensitivity of $100 \%$ in an observational case-control study [23]. Another study indicated that a full LUS thickness of $<2.3 \mathrm{~mm}$ measured between 35 and 38 weeks of gestation is associated with a higher risk of complete uterine rupture [20]. No uterine dehiscence occurred when the full LUS thickness was more than $4.5 \mathrm{~mm}$ [24]. In the current study, TRM measured using LUS significantly increased from $2.42 \pm 0.1 .04 \mathrm{~mm}$ to $8.36 \pm 3.11 \mathrm{~mm}$, and no cases of threatened uterine rupture or uterine rupture were detected in women who had successful deliveries. This result further demonstrated that vaginal repair has the ability not only to resolve the symptom of prolonged menstrual bleeding but also to rebuild the muscular layer of the scar site.

As reported by Vervoort [25], a number of factors may explain the development of a caesarean scar defect: [1] a very low incision through the cervical tissue; [2] inadequate suturing or incomplete closure of the uterine wall due to a single-layer endometrial-saving closure technique or use of locking sutures; and [3] surgical interventions that encourage adhesion formation (namely, non-closure of the peritoneum, inadequate haemostasis, visible sutures, and others). Our research is the first of its type to detect residual myometrium under the microscope. We found that the collagen density of the residual myometrium covering the scar was significantly higher than that of healthy myometrium directly adjacent to the scar. We believe that the excessive expression of collagen may be an important factor in the formation of diverticula. Thus, the formation of the diverticula may be associated with an imbalance between the ratio of muscle fibres to collagen fibres. Further research will be needed to clarify these issues.

In the current study, 6 of the $20(30.0 \%)$ women who achieved pregnancy without miscarriage experienced pregnancy complications, including 2 women with preterm births, 2 women with GDM, 1 woman with placenta previa, and 1 woman with GDM as well as hypothyroidism during pregnancy. Notably, haemorrhage (945 $\mathrm{ml}$ blood) occurred in the patient who had a preterm birth. The ages of the women and the intervals from vaginal repair to pregnancy may be responsible for the relatively high rate of pregnancy complications.

\section{Conclusion}

The current study demonstrated that vaginal repair can not only resolve CSD-associated prolonged menstrual bleeding but can also restore the anatomical structure in patients with CSD. Furthermore, subsequent satisfactory obstetrical outcomes can be achieved in vaginal repair-treated CSD patients. 


\section{Abbreviations}

CS: Caesarean section; CSD: Caesarean scar diverticula (defect); GDM: Gestational diabetes mellitus; LUS: Lower uterine segment; TRM: Thickness of the remaining muscular layer; TVU: Transvaginal ultrasound

\section{Acknowledgements}

We thank all of the patients, doctors and nurses who participated in this study.

\section{Funding}

This study was supported by a Key grant from the Shanghai Scientific and Technology Commission (15411961600) and a action plan from Shenkang(16CR4028A). Key grant from the Shanghai Scientific and Technology Commission (15411961600): design of the study, collection, analysis. Action plan from Shenkang(16CR4028A): interpretation of data, and in writing the manuscript.

\section{Availability of data and materials}

The datasets used and analysed during the current study are available from the corresponding author on reasonable request.

\section{Authors' contributions}

XZ: Manuscript writing,data analysis. HC: Data collection. XY: Data collection or management. XF: Manuscript revision,data analysis. XW: Project development, manuscript editing. All authors read and approved the final manuscript.

\section{Ethics approval and consent to participate}

This study was approved by the Ethics Committee of Shanghai First Maternity and Infant Hospital (KS1512). Informed written consent was obtained from all participants.

\section{Consent for publication}

Not applicable.

\section{Competing interests}

The results and writing of this study was supported by a Key grant from the Shanghai Scientific and Technology Commission (15411961600) and a action plan from Shenkang(16CR4028A), the authors have no conflicts of interest.

\section{Publisher's Note}

Springer Nature remains neutral with regard to jurisdictional claims in published maps and institutional affiliations.

\section{Author details}

'Department of Gynecology, Xinhua Hospital affiliated with Shanghai Jiaotong University, 1665 Kong Jiang Rd, Yang Pu District, Shanghai 200092, China. ${ }^{2}$ Department of Gynecology, Shanghai First Maternity and Infant Hospital, Affiliated to Tongji University, Shanghai, China.

\section{Received: 10 January 2018 Accepted: 17 September 2018}

\section{Published online: 19 October 2018}

\section{References}

1. Donnez O, Donnez J, Orellana R, Dolmans MM. Gynecological and obstetrical outcomes after laparoscopic repair of a cesarean scar defect in a series of 38 women. Fertil Steril. 2017;107(1):289-96 e282.

2. Vikhareva Osser O, Valentin L. Risk factors for incomplete healing of the uterine incision after caesarean section. BJOG : an international journal of obstetrics and gynaecology. 2010;117(9):1119-26.

3. Osser OV, Jokubkiene L, Valentin L. High prevalence of defects in cesarean section scars at transvaginal ultrasound examination. Ultrasound in obstetrics \& gynecology : the official journal of the International Society of Ultrasound in Obstetrics and Gynecology. 2009;34(1):90-7.

4. van der Voet LF, Vervoort AJ, Veersema S, BijdeVaate AJ, Brolmann HA Huirne JA: Minimally invasive therapy for gynaecological symptoms related to a niche in the caesarean scar: a systematic review. BJOG : an international journal of obstetrics and gynaecology 2014; 121(2):145-156.

5. Chang Y, Tsai EM, Long CY, Lee CL, Kay N. Resectoscopic treatment combined with sonohysterographic evaluation of women with postmenstrual bleeding as a result of previous cesarean delivery scar defects. American journal of obstetrics and gynecology. 2009;200(4):370 e371-4.

6. Bij de Vaate AJ, van der Voet LF, Naji O, Witmer M, Veersema S, Brolmann HA, Bourne T, Huirne JA. Prevalence, potential risk factors for development and symptoms related to the presence of uterine niches following cesarean section: systematic review. Ultrasound in obstetrics \& gynecology : the official journal of the International Society of Ultrasound in Obstetrics and Gynecology. 2014;43(4):372-82.

7. Hager RM, Daltveit AK, Hofoss D, Nilsen ST, Kolaas T, Oian P, Henriksen T. Complications of cesarean deliveries: rates and risk factors. Am J Obstet Gynecol. 2004;190(2):428-34.

8. Ofili-Yebovi D, Ben-Nagi J, Sawyer E, Yazbek J, Lee C, Gonzalez J, Jurkovic D. Deficient lower-segment cesarean section scars: prevalence and risk factors. Ultrasound in obstetrics \& gynecology : the official journal of the International Society of Ultrasound in Obstetrics and Gynecology. 2008:31(1):72-7.

9. Xie H, Wu Y, Yu F, He M, Cao M, Yao S. A comparison of vaginal surgery and operative hysteroscopy for the treatment of cesarean-induced isthmocele: a retrospective review. Gynecol Obstet Investig. 2014;77(2):78-83.

10. Zhou J, Yao M, Wang $H$, Tan W, Chen $P$, Wang $X$. Vaginal repair of cesarean section scar diverticula that resulted in improved postoperative menstruation. J Minim Invasive Gynecol. 2016;23(6):969-78.

11. Zhou X, Yao M, Zhou J, Tan W, Wang H, Wang X. Defect width: the prognostic index for vaginal repair of cesarean section diverticula. Arch Gynecol Obstet. 2017;295(3):623-30.

12. Roberge S, Boutin A, Chaillet N, Moore L, Jastrow N, Demers S, Bujold E. Systematic review of cesarean scar assessment in the nonpregnant state: imaging techniques and uterine scar defect. Am J Perinatol. 2012;29(6):465-71.

13. Vikhareva Osser O, Valentin L. Clinical importance of appearance of cesarean hysterotomy scar at transvaginal ultrasonography in nonpregnant women. Obstet Gynecol. 2011;117(3):525-32.

14. Zhang $X$, Yang M, Wang Q, Chen J, Ding J, Hua K. Prospective evaluation of five methods used to treat cesarean scar defects. International journal of gynaecology and obstetrics: the official organ of the International Federation of Gynaecology and Obstetrics. 2016;134(3):336-9.

15. Luo L, Niu G, Wang Q, Xie HZ, Yao SZ. Vaginal repair of cesarean section scar diverticula. J Minim Invasive Gynecol. 2012;19(4):454-8.

16. Jeremy B, Bonneau C, Guillo E, Paniel BJ, Le Tohic A, Haddad B, Madelenat $P$ : [uterine ishtmique transmural hernia: results of its repair on symptoms and fertility]. Gynecologie, obstetrique \& fertilite 2013; 41(10):588-596.

17. Marotta ML, Donnez J, Squifflet J, Jadoul P, Darii N, Donnez O. Laparoscopic repair of post-cesarean section uterine scar defects diagnosed in nonpregnant women. J Minim Invasive Gynecol. 2013;20(3):386-91.

18. Tanimura S, Funamoto H, Hosono T, Shitano Y, Nakashima M, Ametani $Y$, Nakano T. New diagnostic criteria and operative strategy for cesarean scar syndrome: endoscopic repair for secondary infertility caused by cesarean scar defect. J Obstet Gynaecol Res. 2015:41(9):1363-9.

19. Gurol-Urganci I, Bou-Antoun S, Lim CP, Cromwell DA, Mahmood TA, Templeton A, van der Meulen $\mathrm{JH}$ : Impact of caesarean section on subsequent fertility: a systematic review and meta-analysis. Hum Reprod 2013; 28(7):1943-1952.

20. Bujold E, Jastrow N, Simoneau J, Brunet S, Gauthier RJ. Prediction of complete uterine rupture by sonographic evaluation of the lower uterine segment. American journal of obstetrics and gynecology. 2009;201(3):320 e321-6.

21. Giakoumelou S, Wheelhouse N, Cuschieri K, Entrican G, Howie SE, Horne AW. The role of infection in miscarriage. Hum Reprod Update. 2016;22(1):116-33.

22. O'Neill SM, Kearney PM, Kenny LC, Khashan AS, Henriksen TB, Lutomski JE, Greene RA. Caesarean delivery and subsequent stillbirth or miscarriage: systematic review and meta-analysis. PLoS One. 2013;8(1):e54588.

23. Gizzo S, Zambon A, Saccardi C, Patrelli TS, Di Gangi S, Carrozzini M, Bertocco A, Capobianco G, D'Antona D, Nardelli GB. Effective anatomical and functional status of the lower uterine segment at term: estimating the risk of uterine dehiscence by ultrasound. Fertil Steril. 2013;99(2):496-501.

24. Rozenberg P, Goffinet F, Phillippe HJ, Nisand I. Ultrasonographic measurement of lower uterine segment to assess risk of defects of scarred uterus. Lancet. 1996;347(8997):281-4.

25. Vervoort AJ, Uittenbogaard LB, Hehenkamp WJ, Brolmann HA, Mol BW, Huirne JA. Why do niches develop in caesarean uterine scars? Hypotheses on the aetiology of niche development. Hum Reprod. 2015;30(12):2695-702. 